\title{
Microscopic methods in analysis of submicron phospholipid dispersions
}

MARCIN PŁACZEK

MAGDALENA KOSELA

Department of Pharmaceutical Technology, Medical University of Gdańsk, Poland

Accepted September 21, 2015

Online published January 21, 2016

\begin{abstract}
Microscopy belongs to the group of tests, used in pharmaceutical technology, that despite the lapse of time and the development of new analytical methods, still remain irreplaceable for the characterization of dispersed drug dosage forms (e.g., suspensions and emulsions). To obtain complete description of a specific drug formulation, such as parenteral colloidal products, a combination of different microscopic techniques is sometimes required. Electron microscopy methods are the most useful ones; however, even such basic methods as optical microscopy may be helpful for determination of some properties of a sample. The publication explicates the most popular microscopical techniques used nowadays for characterization of the morphology of nanoparticles suspended in pharmaceutical formulations; advantages and disadvantages of these methods are also discussed. Parenteral submicron formulations containing lecithin or a particular phospholipid were chosen as examples.
\end{abstract}

Keywords: optical microscopy, electron microscopy, AFM, phospholipid dispersions, parenteral drug delivery systems

\section{Phospholipid nanoparticles as parenteral drug delivery carriers}

Nanotechnology is currently one of the most rapidly developing branches of science, encompassing different fields of research, including pharmaceutical sciences. Nanoparticles utilized in drug delivery systems are complex structures that generally do not exceed 200-500 $\mathrm{nm}$ in size. Depending on the production process, these nanoparticles can take the form of nanospheres (matrix-type nanodevices), nanocapsules - in their structure a core and shell can be distinguished (reservoir-type nanodevices) or nanosized emulsions (1-5).

A special role in the development of modern, parenteral, submicron drug forms used in therapy is played by phospholipids which, for pharmaceutical purposes, are mainly obtained from lecithin. As one of the few excipients used in pharmaceutical technology,

\footnotetext{
*Correspondence; e-mail: mpl@gumed.edu.pl
} 
lecithin is regarded as a safe ingredient when applied intravenously and is well-tolerated by patients, even during long-term therapy (6-8). On an industrial scale, lecithin phospholipids have been used for years for the production of submicron emulsions, liposomal dispersions and mixed micelle formulations $(9,10)$. Furthermore, attempts are made to employ lecithin in the formulation of new, parenteral drug carriers. Tests are in progress on such systems as solid lipid nanoparticles (SLN) $(11,12)$, vesicular phospholipid gels (VPGs) (13-15) and aqueous lecithin dispersions (WLD) (16-18).

Advancements in nanotechnology of drug-delivery carriers have led to the development of modern techniques used to characterize their properties, in particular, shape, size, surface and structure. Such properties can be studied by means of numerous instrumental methods such as photon correlation spectroscopy (PCS), laser diffraction (LD), intensity light scattering (ILS), electrophoretic light scattering (ELS), field-flow fractionation, hydrodynamic chromatography, capillary zone electrophoresis, nuclear magnetic resonance spectroscopy, Fourier transform infrared spectroscopy and finally microscopic techniques $(19,20)$.

\section{Microscopic techniques for visualization of phospholipid drug carriers}

A review of the literature indicates that microscopic methods are a valuable tool for the evaluation of various drug forms, including the parenteral forms created with the use

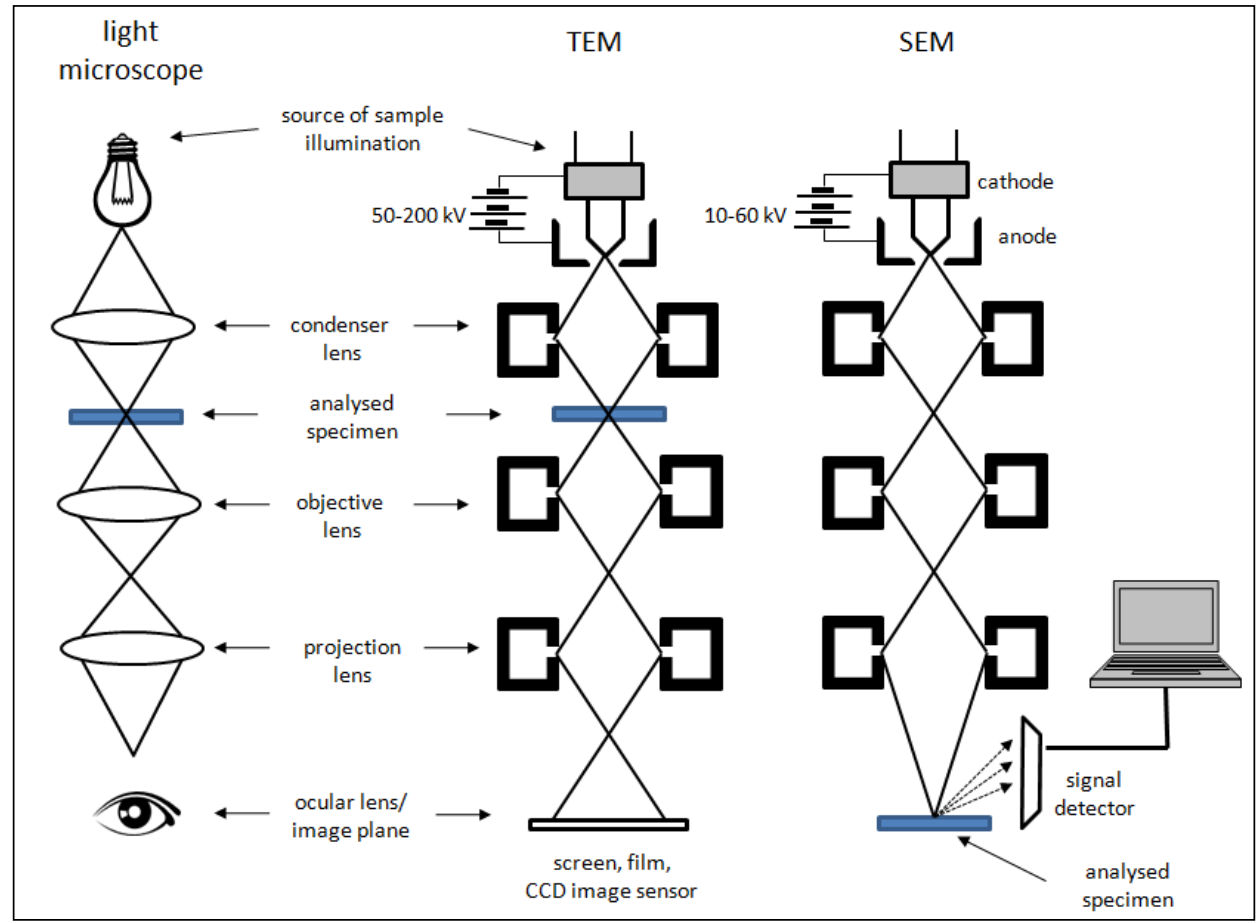

Fig. 1. Similarities and differences in image formation between light and electron microscopes $(23,25$, modified). 


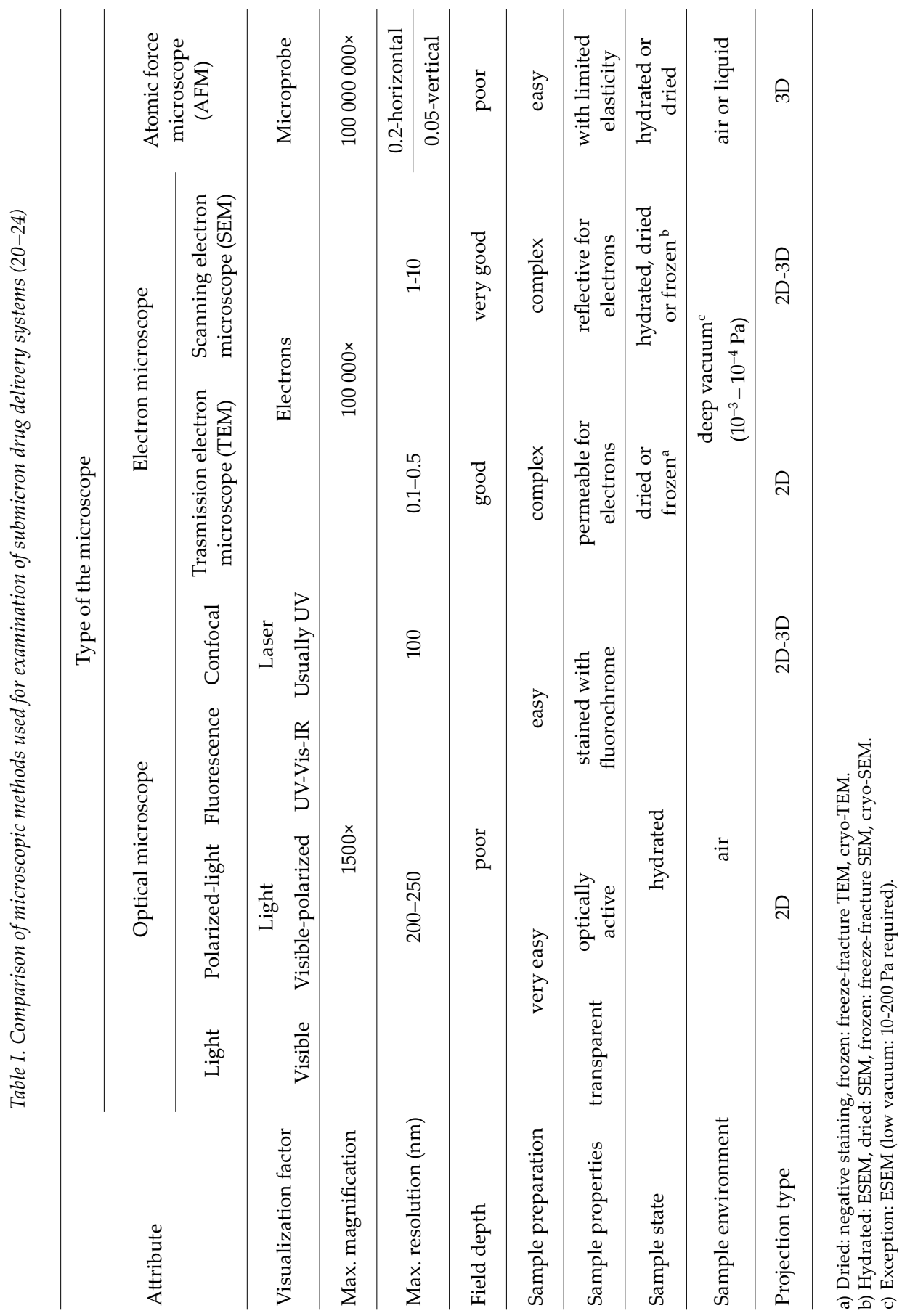


of phospholipids. The simplest method, frequently used for initial testing, is optical light microscopy and its various modifications, while electron microscopy and atomic force microscopy are used when more detailed information is required (20-22). Each of these methods requires sample preparation in a specific way, depending on the degree of complexity, and each involves different capabilities and certain limitations, e.g., the risk of error or artifact formation. Below, we give an overview of different microscopic techniques used for the visualization of dispersion systems, with special reference to phospholipid containing formulations. The benefits and drawbacks of the methods are indicated. Data contained in Table I constitutes an introduction to the comparative analysis of microscopic methods, while the more detailed description was divided into specific subsections, from light (optical) microscopy, electron microscopy to atomic force microscopy.

Light microscopy. - Light (optical) microscope is an instrument that uses a visible electromagnetic wave frequency of 400-700 $\mathrm{nm}$ length. The image obtained by the light microscope is created by means of a set of optical elements (Fig. 1) and total magnification of the sample, obtained by this technique, is usually limited to the range of 10-1500× (23).

Not only magnification has an effect on the quality of the microscopic image, but, above all, the resolution of the optical system, i.e. the value corresponding to the smallest distance that divides two points, which in the microscopic image are perceived separately. According to Ernst Abbe's theory of microscopes (25), the resolution of a microscope is limited by the light wavelength, as expressed by the following equation:

$$
d 0=\frac{0.61 \times \lambda}{N A}[\mathrm{~nm}]
$$

where $d 0$ - resolution, $\lambda$ - light wavelength, $N A$ - numerical aperture of the objective lens.

Cover slip choice, condenser NA and selection of liquid medium also affect the ultimate resolution. However, at best, the resolution of light microscopes is not higher than 0.2 $\mu \mathrm{m}$. Due to method limitations (small magnification and low resolution), the light microscope is used only during the initial stages of testing conducted on submicron systems (22). An indisputable benefit of this method, however, is the apparatus availability, simple and user-friendly use of the microscope and fast preparation of the analyzed samples.

Fig. 2 presents an image obtained by means of the light microscopy technique, showing various parenteral preparations containing phospholipids: submicron emulsion, solid lipid nanoparticles, liposomes and WLD dispersion.

The presented image indicates that the analysis of dispersion systems by means of an optical microscope can be used to preliminarily define particle size and sample homogeneity rather than to evaluate the morphology and internal structure of individual lipid particles. In the literature $(26,27)$, however, one can find information about liposome visualization carried out by means of the light microscopy method, but it applies to large particles (above $500 \mathrm{~nm}$ ) with complex structure only, e.g. giant unilamellar vesicles (GUV) or multilamellar vesicles (MLV). In the case of preparations containing particles smaller in size, this method is limited to detection of pronounced destabilization phenomena such as coalescence (submicron emulsion) or the formation of larger particle aggregates (SLN, WLD or liposomes dispersion). To obtain a more detailed analysis of the preparation and more complete information about the studied structures, it is possible to combine the light microscope with a polarizing or fluorescence microscope into a single apparatus (23). 


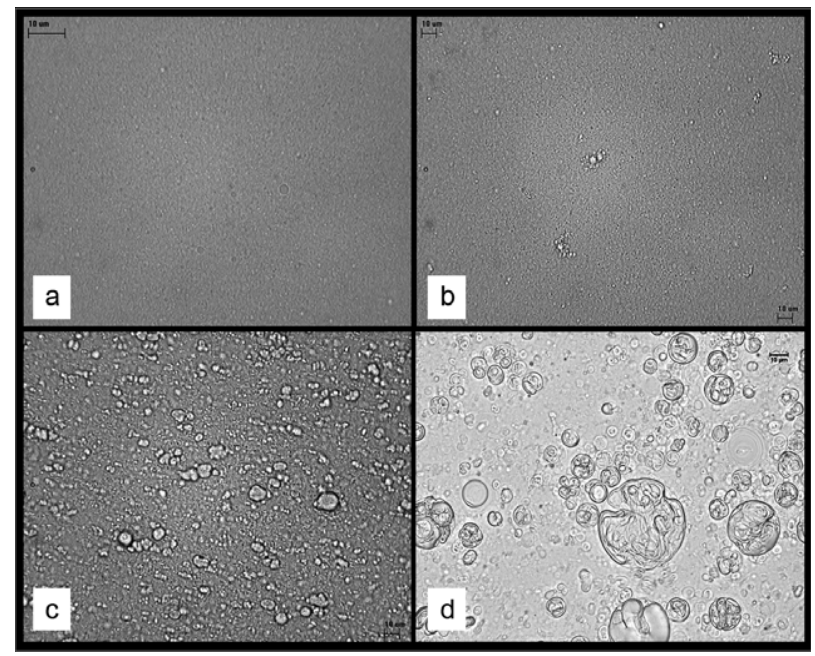

Fig. 2. Light microscopy; images of parenteral dispersed systems containing phospholipids: a) submicron emulsion, b) SLN dispersion, c) liposomes and d) WLD formulation. Bar - $10 \mu \mathrm{m}$.

Polarized light microscopy. - Polarization microscopy can be employed, among others, in the analysis of parenteral dispersion systems to confirm the presence of liposomes or similar structures (WLD, vesicles in VPGs) in the studied formulation (Fig. 3). Their anisotropic properties result from the ordered, concentric arrangement of phospholipid layers $(22,28,29)$. Once the polarized rays pass through the lamellar layers, arranged perpendicularly to the polarization plane, the so called "maltese cross" textures appear in the microscopic image. The obtained image is black and white (Fig. 3b, $d$, e and f). If, however, undulation is applied (a birefringent plate located in the way of polarized rays), it is possible to obtain additive interference colours, which appear more vivid, such as first-order, blue, yellow and red (Fig. 3c). Polarization microscopy is also used to detect the liposomes present in emulsion systems, because oil drops, in contrast to phospholipid vesicles, do not possess optically active properties (30).

Fluorescence microscopy. - The phenomenon of fluorescence can be used in the microscopic analysis of colloidal systems. Submicron drug carriers do not demonstrate such ability on their own (they are not capable of auto-fluorescence); this is why lipophilic fluorochromes (Sudan Black B, Oil Red O), as well as hydrophilic ones (sodium fluorescein, fluorescein isothiocyanate, carboxyfluorescein) are used for visualization (23). The image obtained by means of a fluorescence microscope is always a negative of the image from a standard light microscope: fluorescent objects appear on a dark background $(22,23)$.

Some authors $(31,32)$ describe the method of using fluorescence microscopy for the visualization of MLV and GUV, in which both the lipophilic structures and aqueous spaces can be stained. Our studies indicate that it is also possible to employ this visualization technique for the analysis of WLD dispersion, mainly in the initial stages of production (prior to homogenization), when lecithin particles create large multilamellar structures during the hydration process (Fig. 4a). 


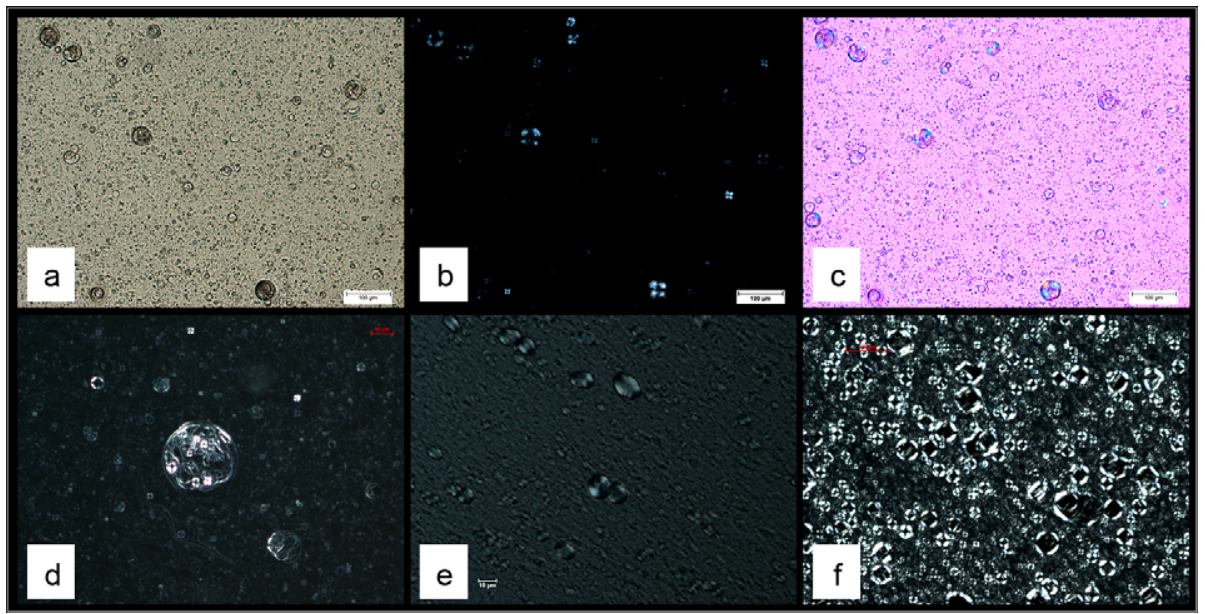

Fig. 3. Polarized-light microscopy; images of WLD dispersion (b, c, d), liposomes (e) and VPGs vesicles (f). Pictures $a, b$ and $c$ show the same formulation of WLD imaged by: $a)$ normal light, $b$ ) polarized light and c) polarized light with lambda plate. Bar -10 or $100 \mu \mathrm{m}$.

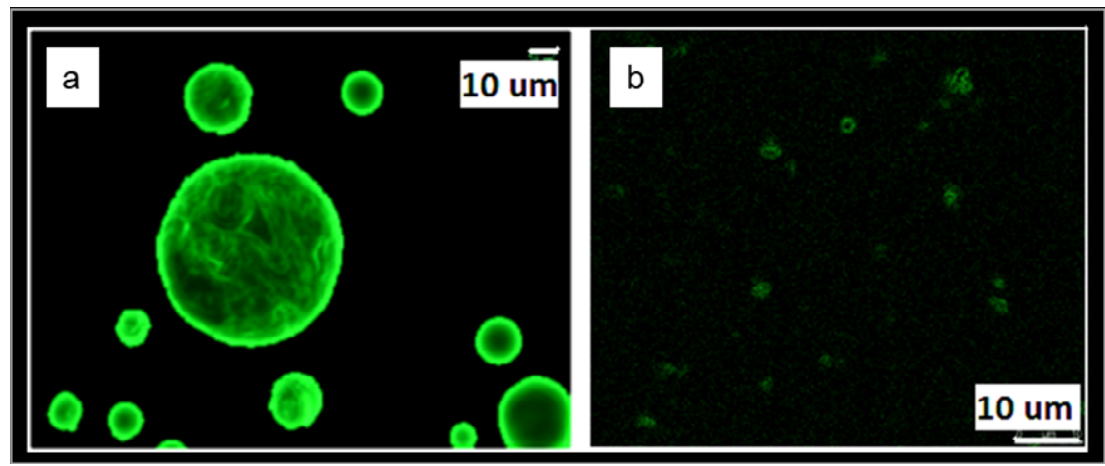

Fig. 4. Lecithin particles dispersed in WLD formulation, stained with sodium fluorescein solution and observed under: a) fluorescence microscope or b) confocal microscope. Bar $-10 \mu \mathrm{m}$.

In the case of GUV, it is possible to conduct an observation detailed enough to detect damage on their surface/coating by means of fluorescence microscopy and the ensuing test results can be used to assess the liposome production methods (31). Also, the degree of discoloration of the formulation provides valuable information about the studied structures, since it can be an indication of differences in the composition of studied carriers, e.g., particles rich in cholesterol withhold more dye (due to their spherical nature) than cholesterol-free particles (polyhedron in structure) (32).

Confocal microscopy. - Fluorescence phenomenon is also employed by a confocal microscope, also known as the confocal laser scanning microscope (CLSM). The light source 
in this case is a UV laser (e.g. Argon gas-laser, emitting different excitation lines in the range of $437-514 \mathrm{~nm}$ ) that illuminates the sample with a light spot of $0.1 \mu \mathrm{m}$ in diameter, which moves quickly while scanning the preparation. This shorter wavelength improves the resolution of the fluorescent image and makes it possible to regulate the intensity of sharpness, so that the preparation surface is scanned at a specific depth. In addition, the sum of sample "optical cross-sections" allows recreating the spatial dimensions of the studied objects. Like in the case of fluorescence microscopy, the samples are stained with fluorochrome and viewed in a hydrated state $(22,23,33)$.

Liposome dispersions are parenteral colloidal systems most frequently studied by means of the CLSM method. Although high resolution ability of an apparatus allows to obtain a good quality image of liposome vesicles, and even to define their complex multilamellar internal structure, one should note that the majority of these type of studies refer to GUV, which can have as many as a dozen or so micrometers in size. Analysis of smaller systems (e.g., WLD formulation) by means of this method is much more problematic, as shown in Fig. $4 b$.

Electron microscopy. - The operation of an electron microscope is based on the same principle that governs the operation of a light microscope, except that electrons are used to illuminate the analyzed sample, instead of light (25). The wavelength of visible light ranges from 400 to $700 \mathrm{~nm}$, while it measures $0.004 \mathrm{~nm}$ for an electron beam. For this reason, when the light wave is replaced by an electron stream, the magnification one can obtain in the electron microscope can reach as much as $100,000 \times$, while the resolution can range from 0.1 to $0.5 \mathrm{~nm}$.

The image-forming system in the electron microscope is analogous to that of the light microscope (Fig. 1). It incorporates the electron stream source (electron gun), which emits the electron stream at high density, forms it into a beam and accelerates it to working velocity $(21,23)$. Electromagnetic lenses, which are the structural elements of the electron microscope, focus the electron beam just like glass lenses in the light microscope (Fig. 1). Since electrons are easily deflected by gas molecules, a high vacuum must be maintained in the electron column, within the range of $10^{-3}-10^{-4} \mathrm{~Pa}\left(10^{-5}-10^{-6} \mathrm{mbar}\right)$. The image formed in the electron microscope is invisible; therefore, it must be projected onto a screen covered with the so called luminophore (a substance emitting visible light under electron bombardment) or a photographic emulsion or is processed electronically and displayed on a monitor (23).

Transmission electron microscopy (TEM). - The transmission electron microscope is an indispensable tool for imaging nanostructures present, among others, in pharmaceutical dispersion formulations. This is possible due to the microscope's high resolution power, which might be limited by the sample properties rather than by the equipment capabilities. In the transmission electron microscope the image is created by electrons, which have passed through the sample-transmitted primary electrons and scattered primary electrons $(20,21,23)$.

TEM - negative staining method. - An important element of TEM analysis is sample preparation. It is, in general, a multiple-stage process requiring great diligence, since it might be a source of artifacts and errors, which may affect image quality and the reliability of conclusions drawn from it. The most commonly used preparation method for the 
evaluation of colloidal formulations is the negative staining method $(20-23,34)$. Electrons are weakly deflected by the components of submicron dispersion and this is the reason why the obtained image is characterized by low contrast. The largest electron deflection is triggered by heavy metals (lead, osmium, wolfram, uranium, molybdenum, platinum), namely, by elements with a high atomic number. For that reason, preparations tested with TEM are "stained" by means of such compounds as uranyl acetate, osmic acid anhydride or ammonium molybdate. These substances have few negative effects on the sample, while, after drying, should form a thin, uniform layer on the sample surface. Staining is performed by placing a drop of the sample on a copper grid (in some cases, the grid is gold or carbon-coated), while the excess is removed by blotting with filter paper. Next, the dye solution is added; after 2 min, it should be rinsed with water and air-dried. When ready, the preparation should be moved to the microscope chamber.

Negative staining can be used, among others, to visualize the size, shape and internal structure of a sample and this is why it is widely used for imaging parenteral pharmaceutical preparations, including those containing phospholipids: submicron emulsions, liposomes or WLD dispersions (Fig. 5).

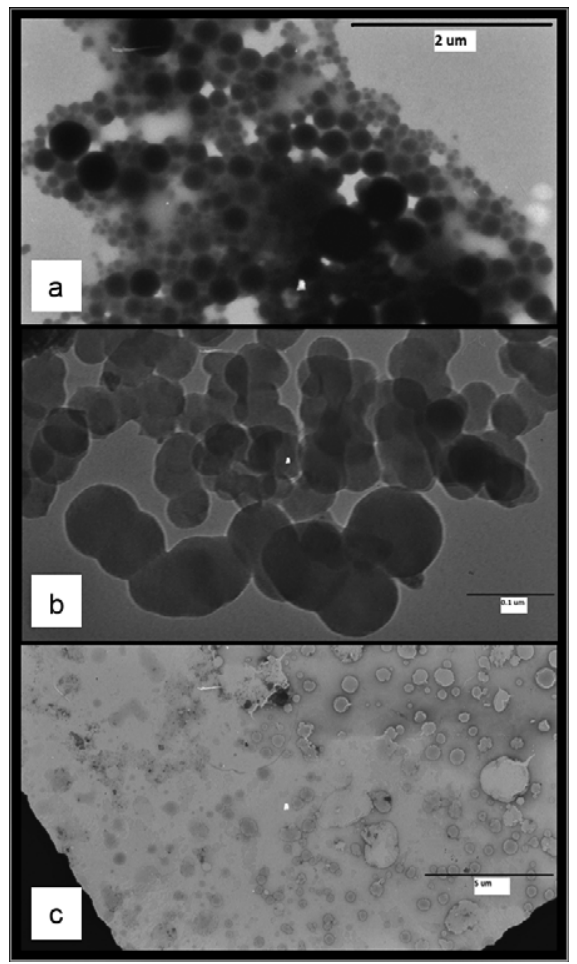

Fig. 5. Electron micrographs (TEM) obtained after negative staining with uranyl acetate of: a) a submicron parenteral emulsion and b) WLD dispersion; c) image demonstrates lecithin particles in WLD formulation damaged during inappropriate staining procedure. Bar: $2 \mu \mathrm{m}, 0.1 \mu \mathrm{m}$ or $1 \mu \mathrm{m}$, respectively. 
It is not easy to obtain an image of good quality using the negative-staining method, while the key-stage in this technique is the right selection of a dye (in terms of type and quality). For instance, while analyzing systems containing phospholipids, it is recommended to cation-stain the sample with uranyl ion (35). The cation binds with the phosphate group of phospholipids, facilitating the analysis of structures (e.g., lipid bilayers) formed in the aqueous environment.

The use of dyes, however, may affect the sample structure, while the staining may be non-uniform (20-22). If some of the sample particles are stained correctly, while others remain invisible, the assessment of system homogeneity is unreliable. Caution, regarding conclusions drawn about the poly-dispersion of a sample or its contamination by other particle structures, should also be taken because of the fact that a very small fragment of a sample is subject to analysis. During staining, light or dark halos can form around some of the structures (e.g., liposomes) and can be incorrectly interpreted as the system of additional lamellar layers. After staining, during the drying phase, a sample may get damaged by surface tension forces or due to the increase of electrolyte concentration. Changes in particle morphology may be also caused by high vacuum present in the microscope chamber, which contributes to sample drying, and by the damaging effect of the electron beam. Fig. $5 \mathrm{c}$ shows the negative consequences of an incorrectly conducted staining process.

Freeze-fracture TEM. - To improve the quality of a microscopic image in TEM analysis, one can employ the sample preparation method known as freeze-fracture. The application of this method allows eliminating the drying phase from the process $(20-22,28,36)$. In this method, the sample preparation process begins with fast-freezing (using liquid propane cooled by liquid nitrogen) of a thin sample layer fitted between gold plates. The next phase involves fracturing of the sample, carried out under vacuum at temperature of approximately $-100{ }^{\circ} \mathrm{C}$. After breakage disclosure, the sublimation process takes place on the ice surface and the deep-seated sample layers become more visible. Next, the cold-fractured surface is coated with an approx. 2-nm deep layer of platinum or gold. Metal sputtering at a specific angle is a process known as "shadowing"; it allows increasing the contrast of the microscopic image. A slightly thicker $(20-30 \mathrm{~nm})$ carbon layer is deposited on the metal layer as it is "transparent to electrons", and its role is to strengthen the examined structure. Next, the specimen is returned to ambient temperature and subjected to chemical etching with chromic or sulphuric acid to remove the sample residue. A ready-made replica is carefully placed on the metal grid and then investigated in TEM (since it is durable, it can be stored and reused for analysis).

The freeze-fracture method employed for liposome imaging allows assessing, in detail, the bilayers present in such phospholipid systems, especially regarding multilamellar vesicles (Fig. 6a). In the case of small unilamellar vesicles, the probability that the internal lamella will be disclosed is much smaller. The sample is usually fractured through the core of the hydrophobic bilayer. As a result, the planar view of the internal sample structure is obtained, allowing assessing the effect that the particular phases of liposome production have on their structure $(20-22,29)$. The freeze-fracture method is also used with great success for the visualization of emulsion systems, since it allows assessing the droplet size (Fig. 6c) and detecting the presence of liposomes and coalescence phenomenon in emulsion systems $(22,37)$. Good results are also obtained for SLN visualization (Fig. 6d).

High resolution is an indisputable advantage of this method. In comparison with other methods, it can be obtained by applying higher electron doses. A drawback of this 


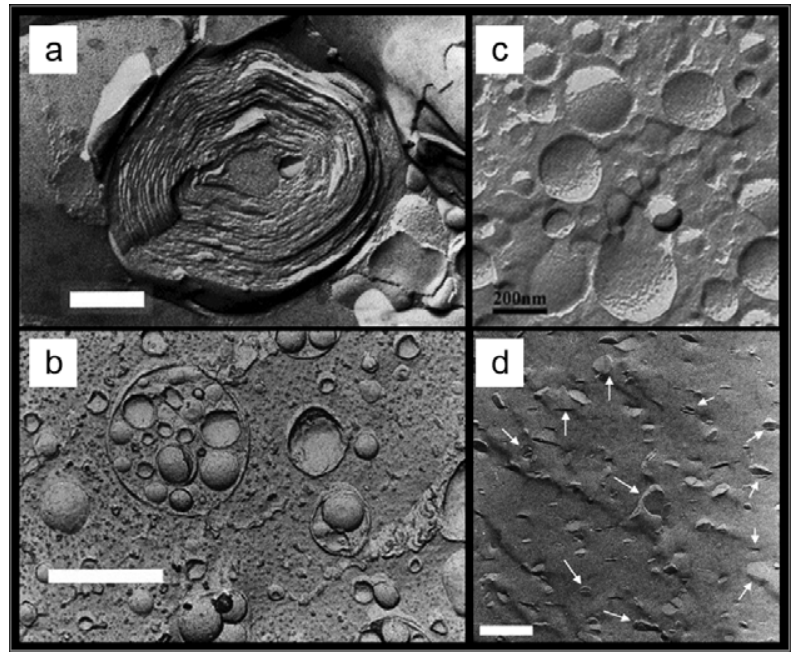

Fig. 6. Freeze-fracture TEM microscopy: a) multilamellar liposome, b) multivesicular liposome, c) submicron emulsion and d) SLN. Reprinted from refs. 29, 36, 37, with permission of Francis group, Elsevier and Springer Open. Bar: $200 \mathrm{~nm}$ (a and c), $1 \mu \mathrm{m}$ (b), or $250 \mathrm{~nm}$ (d).

method is, however, a multi-phase character of the preparation process and the subsequent risk of making an error at one of the phases, e.g., insufficient sample freezing, adsorption of solvent molecules on the sample surface or mechanical damage.

Cryo-TEM analysis. - Sample fixation by freezing is also employed in the cryo-TEM method, considered nowadays as one of the best methods for colloidal drug carrier characterization. Preparation of a good quality formulation is a multi-phase procedure (20-23). A drop of sample is applied to a copper grid covered with a porous carbon layer. The excess sample is removed by quick blotting with filter paper leaving a film of approx. $100 \mathrm{~nm}$ thickness, which fills up the holes in the carbon layer. Next, the sample is plunged-frozen in liquid ethane, cooled by liquid nitrogen. This process should be conducted quickly to avoid the formation of crystalline ice. In an ideal situation, water is vitrified, i.e., transformed into a glassy state, where ice has an amorphous structure. After freezing, the sample needs to be kept at the temperature of approx. $-175{ }^{\circ} \mathrm{C}$ (in order to avoid the phase transition of vitrified water into crystalline ice). For that reason, the sample is placed in a special, liquid nitrogen-cooled holder, which allows to transfer the sample into an electron microscope. Automatic systems are now used for sample freezing, since they are recommended due to improved repeatability (the obtained preparations have the same thickness) and the possibility to control temperature and humidity conditions during the entire process. To make sample analysis by means of the cryo-TEM method possible, the sample has to demonstrate the following characteristics:

(i) the particle size should be within the submicron range, since larger particles will be removed at the drying phase, along with the excess sample, by quick blotting with filter paper, 
(ii) the sample should not be characterized by excessively high viscosity, since difficulties may arise when making the preparation a uniform layer,

(iii) highly concentrated samples should be diluted prior to analysis, to make sure that particles seen in the microscopic image are not densely concentrated $(0.1-0.2 \%$ is the recommended sample concentration).

Cryo-TEM method is commonly employed to assess the shape, size and internal structure of such drug carriers as emulsions, liposomes and solid lipid nanoparticles (Fig. 7). This method can be also used for the analysis of WLD systems. In the case of liposomes and WLD, the method allows getting a relatively good contrast of the lipid bilayer; this is why it is frequently used for thickness and structure evaluation (Fig. 7b,c). Moreover, it is relatively easy to distinguish liposomes from the micelles, that coexist in the studied system (28).

In some cases, it is possible to visualize the surfaces of modified liposomes. Although, due to an overly low contrast of macrogol, it is not possible to use this technique to assess the PEGylated liposomes, one can use it to visualize liposomes (the so called „layersomes”) coated with polylysine or polyglutamic acid (Fig. 7e) (21, 39).

Cryo-TEM method can be also used to evaluate the process of encapsulating a drug substance into liposomes. Such tests were conducted, for instance, with doxorubicin, idarubicin, topotecan, vinorelbin and vincristin. Drug precipitates were visible in the microscopic image as discrete structures in the liposome core or as an increase in contrast of the liposome core, when the drug precipitated in amorphous form (Fig. 7d) (21, 38).

In the image obtained by the cryo-TEM method, one can observe droplets of various size and contrast during visualization of the emulsion sample (Fig. 7a). It is also possible to

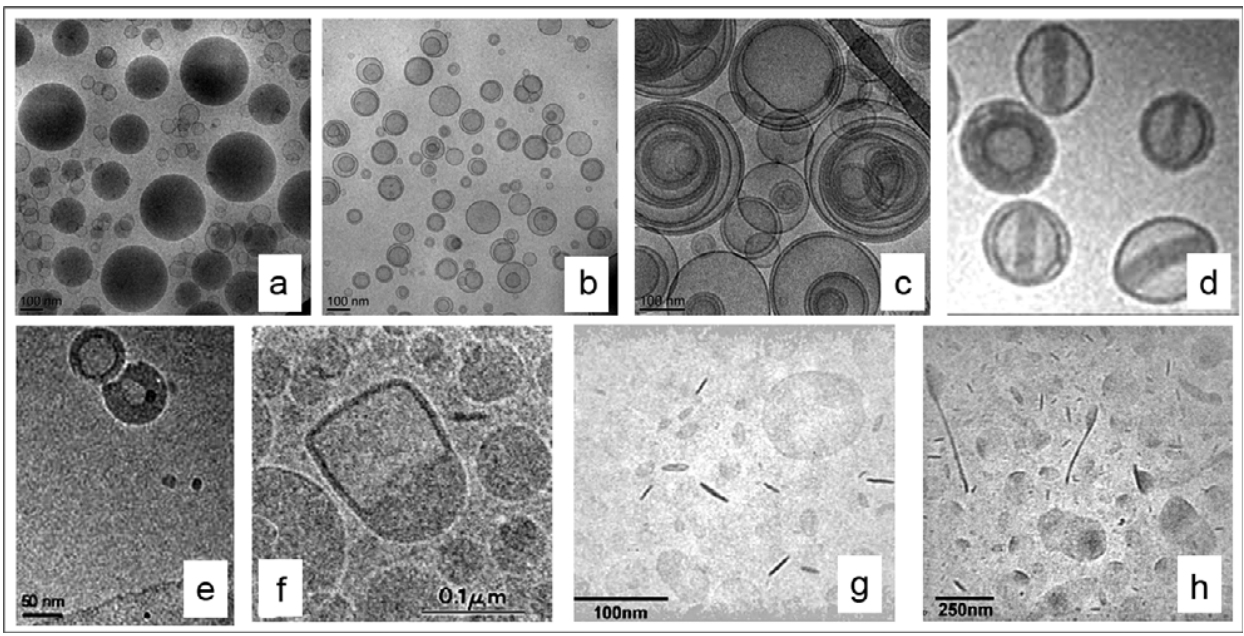

Fig. 7. Phospholipid formulations analyzed by the cryo-TEM technique: a) submicron emulsion, b) WLD dispersion, c) multilamellar and multivesicular liposomes, d) drug containing liposomes, e) coated liposomes-layersomes, f) emulsion with stearylamine, g) SLN and h) NLC. Pictures d-h reprinted from refs. 38-41, with permission of Elsevier and Springer. Bar: $50 \mathrm{~nm}(\mathrm{e}), 100 \mathrm{~nm}(\mathrm{a}-\mathrm{d}, \mathrm{f}, \mathrm{g})$ and $250 \mathrm{~nm}(\mathrm{~h})$. 
visualize the effect the excipients have on the morphology of emulsion droplets, e.g., after the addition of stearylamine to the emulsion composed of MCT oil and purified egg yolk lecithin, one can observe a "handbag"-like structures in the cryo-TEM images (Fig. 7f) (40).

Application of the cryo-TEM method helped elucidate a number of important structural characteristics of the SLN system. It was demonstrated, among others, that lipid nanoparticles visualized in the microscopic image can be of different shape (circular, ellipsoidal or elongated), while their appearance changes according to the viewing angle (Fig. 7g) (20, 21, 41). Moreover, precise visualization of the systems containing solid lipid nanoparticles, with liquid oil in their composition, better known as nanostructured lipid carriers (NLC), allows observing oil droplets attached to the surface of solid lipids (Fig. 7h).

Despite a number of benefits, the cryo-TEM method is not free of certain limitations and, like every microscopic method, is composed of a number of critical phases, regarding image quality. It may prove difficult to get a glassy ice layer at a specific thickness. The layer must be sufficiently thin to be suitable for microscopic analysis, but also thick enough not to affect the sample structure. It is extremely important to continuously chill the sample during analysis, since changes in temperature and humidity may cause damage to the preparation. Despite fixing, the sample remains susceptible to the damaging effect of the electron beam, manifested, e.g., as air bubbles in the preparation. This limits the sample viewing time and reduces the size of the applied electron doses. It may also happen that a non-uniform particle distribution is observed in the microscopic image. Larger particles band together in the thicker zone of the sample (close to the wire of the grid used as preparation carrier) are more susceptible to the damaging effect of the electrons (in consequence, they may remain undetected), while smaller ones move to the thinner layer $(22,21,28)$.

To assess the particle size distribution, one needs to analyze a larger number of individual images and use appropriate software. Despite the fact that some phases of the cryo-TEM method are automated, sample preparation is costly and time-consuming. Therefore, despite the increasing availability of this method for the colloidal drug carrier analysis, it is not used for the routine quality control of submicron preparations.

Scanning electron microscopy (SEM). - Scanning electron microscopy is used for studying sample surfaces and allows obtaining images with a great field depth. It differs from the transmission microscope in its construction and principles of operation (Fig. 1). The electron gun emits a concentrated electron beam that scans the sample surface coated with a metal layer during the preparation process. The metallic coat does not release electrons but emits some of the secondary electrons, which, along with the backscattered electrons, carry crucial information for image generation. The acceleration voltage for the scanning microscope measures $10-60 \mathrm{kV}$; it is lower, therefore, than the voltage used in TEM. Unfortunately, resolution is also weaker and measures 1-10 nm $(20,23)$. Only the recently developed scanning microscopes with a modified electron gun (field emission scanning electron microscopes, FE-SEM) and additional detector allow obtaining a resolution power comparable to the resolution of the transmission microscope $(42,43)$. Scanning microscopy does not furnish information about the internal structure of studied objects; its advantage, however, is that it allows assessing sample topography (quasi 3D projection) in contrast to two-dimensional images obtained by standard TEM (20).

Due to the conditions present inside the SEM microscope (high vacuum: $10^{-3}-10^{-4} \mathrm{~Pa}$ ), one cannot use this method to analyze liquid samples. The preparation methods of dispersion systems for SEM analysis, described in scientific publications, show significant differ- 
ences; one can distinguish, however, two basic phases: water removal from sample (a phase completed by drying at a critical point or freeze-drying) and surface-coating with metal (gold, platinum or palladium alloys) $(20,21,43)$.

In the case of submicron emulsions, such a procedure allows to assess the size of the internal phase and to determine system durability - visualization of coalescence phenomenon. In the case of liposome analysis, however, their dispersion is usually preceded by fixation, whose role is to make the lipid bilayer more "rigid". For that purpose, formaldehyde and glutaraldehyde are used first, followed by osmium tetroxide. If testing is conducted under an FE-SEM microscope, it is possible to use the acceleration voltage at very low values $(2-5 \mathrm{kV})$ to hinder the damaging effect of the electron beam. Microscope resolution allows to conduct a detailed analysis of giant liposome surfaces and of changes in their appearance due to the PEGylation process (the surface of PEGylated liposomes is more irregular in character) (Figs. 8a,b). The method also allows visualizing changes in liposome morphology and assessing their stability in long-term studies and visualizing liposome complexes with proteins and DNA (43).

Cryo-SEM and freeze-fracture SEM analysis. - Like in the case of the TEM method, the preparation of a sample for SEM analysis can include the freezing phase based on the vitrification effect (cryo-SEM) or freeze and fracture (in this case, the last phase involves coating with a metal layer) (Figs. 8c,d) $(20,42,44)$. The cryo-SEM method can be employed to test samples, which, due to high density, susceptibility to aggregate and lack of possibility to form a thin and uniform layer, are not suitable for the analysis by the cryo-TEM method. Furthermore, by cryo-SEM gives more comprehensive information about nonhomogenous samples, since a larger area of the preparation is studied. Using of cryo-FE-

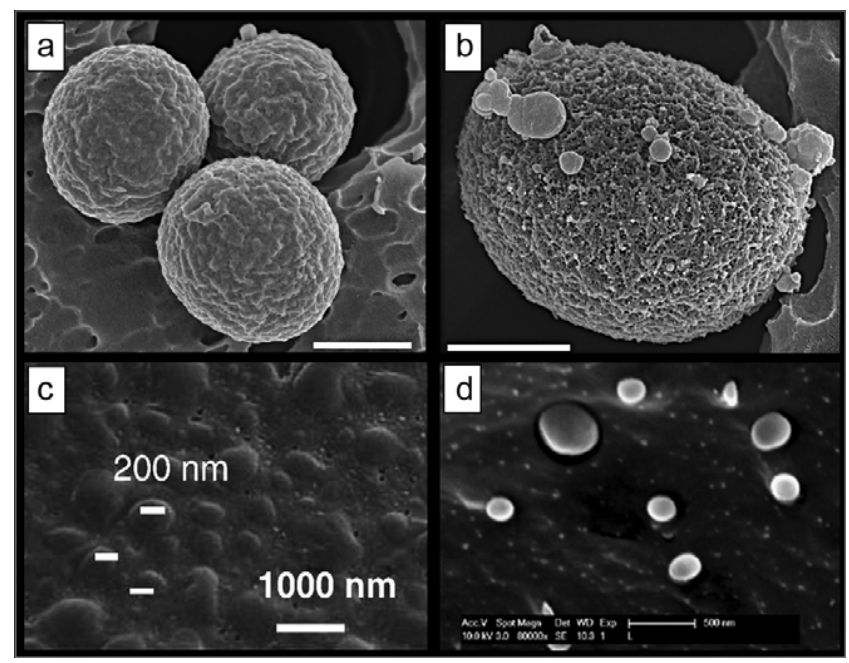

Fig. 8. Images obtained by the FE-SEM method: a) giant, non-PEGylated and b) PEGylated liposome, cryo-FE-SEM method, c) submicron emulsion and freeze-fracture SEM method, d) submicron emulsion. Pictures reprinted from refs. 43 (a and b), ref. 44 (c) and ref. 45 (d), with permission of Elsevier and Springer. Bar: $0.5 \mu \mathrm{m}(\mathrm{a}), 1 \mu \mathrm{m}(\mathrm{b}$ and $\mathrm{d})$. 
SEM analysis, it is possible to study emulsions, solid lipid nanoparticles (SLN) and nanostructured lipid carriers (NLC). The method allows determining the shape and size of such particles and excluding the presence of oil droplets in NLC samples (44).

ESEM and wet STEM techniques. - Environmental scanning electron microscopy (ESEM) is a recent advancement for electron microscopical interrogation of labile and biological specimens, primarily through conservation of specimen water by a partial pressure environment. This control allows imaging of hydrated samples such as colloidal drug carriers in a less-invasive manner, without preliminary sample preparation, in a more "natural" environment, owing to the application of a differential pressure system, which isolates the electron column (high vacuum) from the sample chamber (low vacuum) $(20,22,43,46)$. In this case, the hydrated preparation is a source of vapour that participates in the creation process of the microscopic image. Secondary and backscattered electrons cause ionization of vapour molecules, generating secondary electrons. Newly-formed electrons can cause ionization of vapour molecules that follow, triggering a cascading reaction and strengthening the detector-reaching signal. The preparation can be viewed in the presence of water vapour or other gases.

With the ESEM method, one can not only obtain information about the shape, size, homogeneity and structure (Fig. 9) of emulsions, liposomes, WLD dispersions, niosomes, liposome complexes with nucleic acids, but also study parameter changes in real time (20, 22,46 ). Furthermore, it is possible to visualize the liposome and niosome formation process and assess their stability; for instance, it was demonstrated that the drug containing nanostructures are more stable and resistant to dehydration than „empty" liposomes or niosomes.

Applying a special unit composed of a Peltier module and a support for the grid, upon which the tested sample is placed (similar to TEM method), the ESEM microscope can analyze the particles contained within the entire volume of the preparation and not only on its surface. This method is known as the wet-scanning transmission electron microscopy. Thanks to this method, the particles suspended in the preparation are immobilized, which makes it possible to assess their properties and determine their surface structure (47). Images of submicron emulsion and WLD, obtained by the wet STEM method, are given in Figs. 9b,c.

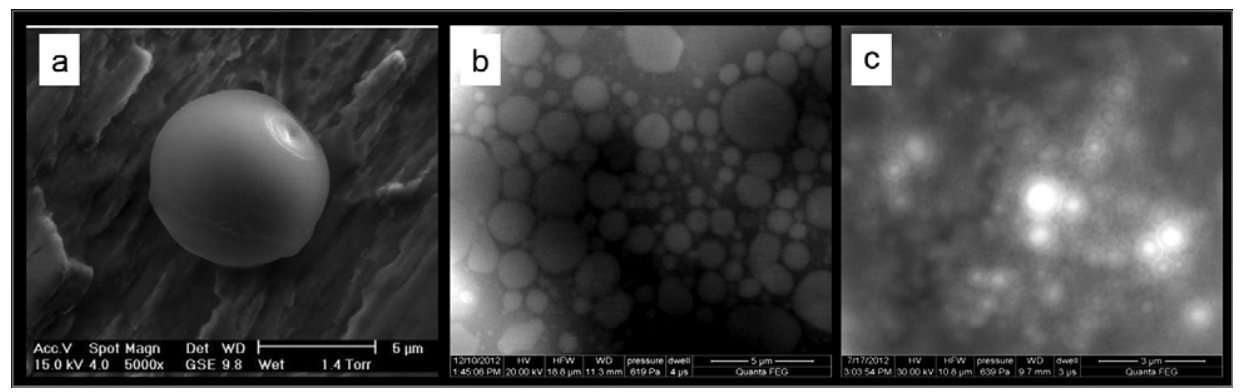

Fig. 9. a) Liposome micrograph prepared with the ESEM technique (reprinted from ref. 22, with permission of Elsevier), “a b) submicron emulsion and c) WLD dispersion analyzed by the wet-STEM method. Bar: $5 \mu \mathrm{m}$ (a and b) or $3 \mu \mathrm{m}$ (c). 
Atomic force microscopy (AFM). - Atomic force microscope is one of the most frequently used microscope devices provided with a scanning probe (SPM) that have revolutionized the technical ability to characterize nanostructures. Such equipment allows to analyze samples (in their dry or hydrated state) in three dimensions, at a sub-nanometer resolution. The sample is scanned by a microprobe (the blade of 5-10 $\mathrm{nm}$ in diameter, fitted to a cantilever), whose deflections are recorded by a laser and photodetector.

The sample is most frequently scanned in two modes:

- contact mode - the blade moves over the preparation surface,

- tapping mode - records changes in the amplitude of microprobe vibrations, triggered upon contact with a sample.

Atomic force microscopy is ideal for a non-invasive visualization of submicron drug carriers, since the sample does not require fixing by freezing or staining $(43,48)$. A drop of the tested dispersion is placed on freshly-cut mica and left to dry at room temperature. The sample prepared in this way and viewed using the AFM method, on the basis of a threedimensional image that also allows measuring particle size, can be used, e.g., to assess the liposome acquisition method (Fig. 10) (49). As studies demonstrate (35), the same liposome systems subjected to confocal microscope analysis showed smaller particle size. The reason for this difference was the adhesion of liposomes to the mica surface and the running of AFM test in ambient air, in contrast to hydrated sample testing performed by means of the confocal microscope.

High resolution used for liposome visualization allows studing in detail the lipid vesicles used as nucleic acid carriers. The use of AFM makes it possible to visualize DNA strand structures, the degree of their condensation and their interaction with liposomes or other particle components, e.g., polymers (49). No other microscopic method allows examining samples in a non-destructive way with such a high degree of resolution.

Aside from information about sample topography, it is also possible to use AFM to study the mechanical properties of submicron particles. This method can be employed, among others, for the evaluation of surface elasticity during the testing of liposome stability. In this case, the microprobe of the microscope, while moving over the studied sample also moves up and down creating, as a result, a map of the preparation elastic properties. By means of this technique, it was demonstrated that cholesterol applied to a liposome composition increases its elasticity several-fold, in contrast to vesicles obtained from egg yolk phosphatidylcholine only (50).

The AFM microscope can be also used to evaluate the nanocapsule formation process, since the apparatus allows distinguishing emulsion droplets (which nanocapsules are made of) from ready-made nanocapsules. As shown in the study (51), it was also possible to determine the number of coating layers formed on nanocapsules and their rigidity due to the equipment's high level of precision.

\section{Microscopy as a supporting technique for instrumental methods dedicated to particles size measurement}

Various instrumental methods are used to determine the size of particles in parenteral products. The size of dispersed particles is important not only from the technological and biopharmaceutical points of view, but it is an important parameter because of patient 

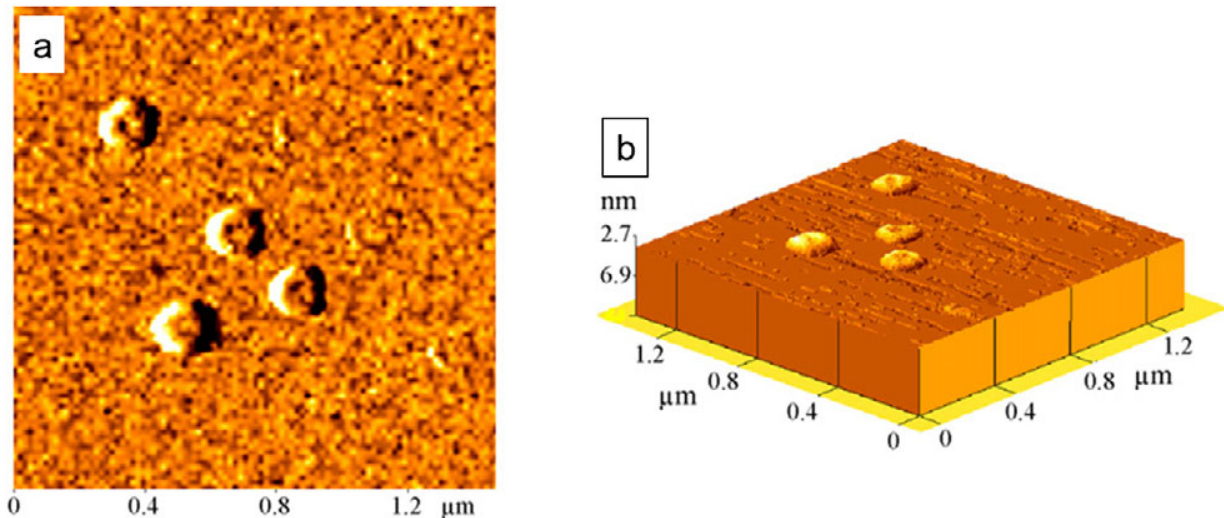

Fig. 10. AFM image of liposomes prepared in hydrated state by non-contact mode: a) topography and b) 3 D-view. Reprinted from ref. 49, with permission of Elsevier.

safety when the product is intended for intravenous administration. The presence of subvisible particles (sized 1-50 $\mu \mathrm{m}$ ) in parenteral fluids may cause adverse reactions such as vein irritation, phlebitis, pulmonary granulomas, local tissue infarction, occlusion of capillaries and arteries, anaphylactic shock or even death $(52,53)$. Current pharmacopoeias contain monographs that describe methods for testing different types of particles: either specific for a certain drug product (e.g., injectable emulsions or liposome dispersions) or for detecting insoluble particles regarded as particulate contamination.

Apart from microscopic analysis, methods based on electronic measurement are proposed for determination of the particle size. The most popular are: (i) light obscuration particle count test; (ii) light diffraction techniques - laser diffraction test (LD) and (iii) dynamic light scattering (DLS). The latter two are currently most frequently employed because of the ease of sample preparation, fast measurement procedure and possible simultaneous determination of the population of particles $(19,54,55)$. Such methods provide valuable information about the average particle size (D [4,3] or Z-average in the LD and DLS technique, respectively) and particle size distribution and homogeneity (span value for LD and polydispersity index - PDI for DLS). However, one should remember that in LD and DLS methods the examined nanocarriers are assumed to be ideal spheres, while in reality the particles may be of a different shape. This simplification may conceal, for example, the presence of larger long particles though they can be responsible for destabilization of the system or possible vascular embolism after intravenous administration. As both techniques cannot distinguish between light scattering or diffraction by a single primary particle and agglomerates, it is also difficult to assess the nature of changes observed in the analyzed samples, e.g., during stability studies. Neither LD nor DLS method allow detecting in the formulation insoluble impurities from foreign bodies, because they are always in a small quantity if expressed as the percentage fraction of the total number of all particles present in dispersed systems. Thus, one should remember that, despite their advantages, these techniques exhibit certain limitations and should not be applied without further (microscopic) verification. 
An example of such problems is demonstrated in detail in Fig. 11 showing the results obtained for one of the commercially available parenteral fat emulsions. Both DLS and LD analyses of the emulsion (Figs. 11a and b) demonstrated that $100 \%$ of the particles were of submicron size: Z-average measured by DLS method was $286 \mathrm{~nm}$ and the volume mean diameter D [4,3] estimated by LD was $330 \mathrm{~nm}$. Size distribution range was narrow and averaged 0.155 (PDI) and 1.28 (span) for DLS and LD tests, respectively.

Microscopic examination of the emulsion performed at the same time has proven, however, that the product was contaminated by particulate matter, sized up to $30 \mu \mathrm{m}$ (Fig. 11c). Moreover, the flake shape of observed particles allowed the conclusion that the plastic container used by the manufacturer as packaging was the source of contamination. Since the number of such fragments was less than $0.01 \%$ of the total number of particles, they were not detected by the DLS and LD tests. Another microscopic method (cryo-TEM) demonstrated a more complex structure of the emulsion, with liposomes present beside oily droplets (Fig. 11d). The size of these two types of particles was similar and the distinction be-
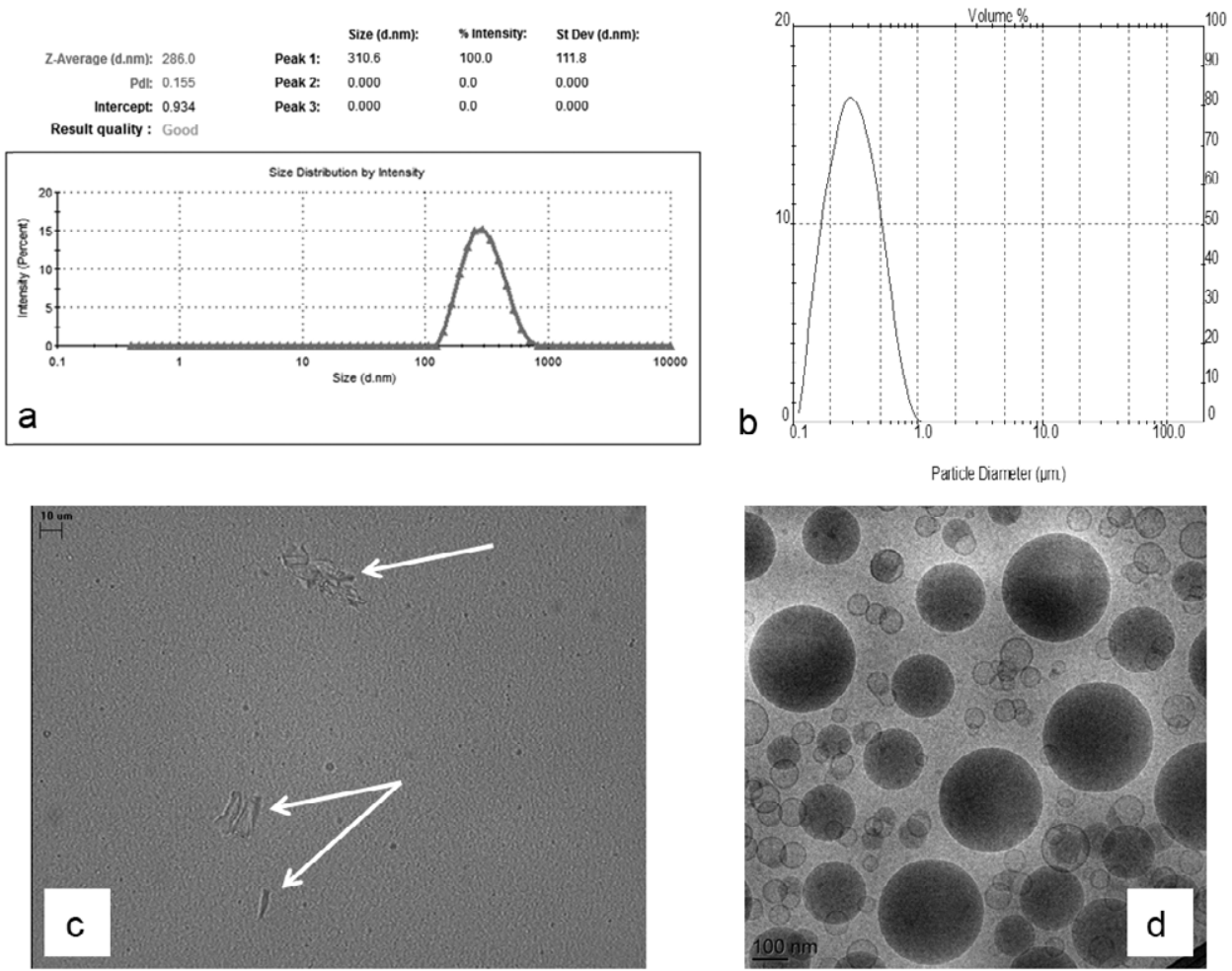

Fig. 11. Characterization of a commercial intravenous fat emulsion using four different analytical techniques: a) DLS, b) LD, c) light microscopy and d) cryo-TEM microscopy. Only the light microscope enabled identification of contamination by particulate matter (arrows), sized up to about $30 \mu \mathrm{m}$ (c) and with the electron microscope two types of nano-sized particles (oily droplets as dark circles, and liposomes as transparent circles) were distinguished (d). 
tween them was impossible without the use of microscopic techniques. This example clearly indicated that additional techniques of particle analysis, such as electron or light microscopy, are highly recommended when studying parenteral drug delivery carriers, even if it is not required by authorities as a part of quality control.

\section{CONCLUSIONS}

In this publication, we have gathered information about the most important methods currently used in qualitative microscopic analysis (light, electron and AFM) of phospholipid parenteral dispersions. Although light microscopy methods are characterized by a rather low resolution not abling one to assess the morphology of particles present in the studied submicron dispersions, they can be definitely used for the preliminary analysis of these systems. With the use of an optical microscope, one can define dispersion homogeneity, the presence of heterogeneous structures (which differ in size or shape in contrast to standard particles), as well as make conclusions about system stability (tendency of the preparation to aggregate or separate is easily observed). An indisputable advantage of this microscopic technique is equipment availability and uncomplicated methodology of analysis. Furthermore, a skilful staining of the preparation by means of an appropriate fluorochrome allows defining certain physicochemical properties of particles and exploring their structure (fluorescence or confocal microscope). If, however, the aim of the study is to define both the morphology of nanoparticles and their complex internal structure, it is necessary to employ one of the techniques of electron microscopy or atomic force microscopy. Microscopic techniques are also very useful for determination of particle size, as an additional and comparative method for electronic measurements (LD or DLS), especially in situations when the type of particles being investigated should be clearly characterized.

In the interest of this study, with regard to its clarity and cohesion, we have purposefully omitted discussion of the techniques of limited use in probing nanoparticles, such as the Electron energy-loss spectroscopy, Energy-filtered transmission electron microscopy, Energydispersive X-ray spectroscopy or AES - Auger electron spectroscopy (AES), belonging to a group of Analytical electron microscopy - for reference, see e.g. ref. 56. Despite the enormous variety of research methods, one should suspect that the intensive growth of nanotechnology in numerous fields of science, non-excluding pharmaceutical technology, will be favorable for the development of subsequent, more advanced microscope visualization techniques.

The overview of different microscopic methods presented in this study allows us to observe differences in the methods of sample preparation for analysis and image creation. Selection of an appropriate method allows obtaining a valuable information about the quality and properties of pharmaceutical products, including systems such as phospholipid, parenteral drug carriers. One should remember, however, that despite their numerous advantages, none of the methods are free of potential defects and errors. This is why it is necessary to combine microscopic methods with other analytical tests $(20,51)$.

Acknowledgments. - This study was financially supported by the National Sciences Center (Cracow, Poland) - grant number N N405 668440. The authors thank Jarosław Korczyński of the Nencki Institute of Experimental Biology of the Polish Academy of Sciences for his assistance in WLD analysis by fluorescence and confocal microscopes. We also thank Dr. Marcin Libera from the Centre of Polymer and Carbon Materials of the Polish Academy of Sciences, Zabrze, for his assistance in analy- 
ses of WLD and submicron emulsions by the wetSTEM technique. Thanks are also due to Dr. Markus Drechsler from the Laboratory for Soft Matter Electron Microscopy, University of Bayreuth, Germany, for excellent collaboration during cryo-TEM analysis. Dorothy and Barbara are kindly acknowledged for drawing our attention to the problem of particulate matter in parenteral emulsion and determining the particle size using DLS and LD methods.

\section{REFERENCES}

1. P. Couvreur, Nanoparticles in drug delivery: past, present and future, Adv. Drug Deliv. Rev. 65 (2013) 21-23; DOI: 10.1016/j.addr.2012.04.010.

2. I. F. Uchegbu and A. Siew, Nanomedicines and nanodiagnostics come of age, J. Pharm. Sci. 102 (2013) 305-310; DOI: 10.1002/jps.23377.

3. T. M. Allen and P. R. Cullis, Liposomal drug delivery systems: from concept to clinical applications, Adv. Drug Deliv. Rev. 65 (2013) 36-48; DOI: 10.1016/j.addr.2012.09.037.

4. E. Fattal and Ch. Vauthier, Drug Delivery: Nanoparticles, in Encyclopedia of Pharmaceutical Technology (Ed. J. Swarbrick), Informa Healthcare, New York 2007, pp. 1183-1200.

5. N. Anton and T. F. Vandamme, Nano-emulsions and micro-emulsions: clarification of the critical differences, Pharm. Res. 28 (2011) 978-985; DOI: 10.1007/s11095-010-0309-1.

6. C. R. Rowe, P. J. Sheskey and S. C. Owen, Handbook of Pharmaceutical Excipients, $5^{\text {th }}$ ed., Pharmaceutical Press, London 2006, pp. 409-411.

7. A. Michajlik and E. Bartnikowska, Lipidy i lipoproteiny osocza, PZWL, Warsaw 1999.

8. W. Bernhard, S. Hoffmann, H. Dombrovsky, G. A. Rau, A. Kamlage, M. Kappler, J. J. Haitsma, J. Freihorst, H. von der Hardt and C. F. Poets, Phosphatidylcholine molecular species in lung surfactant: composition in relation to respiratory rate and lung development, Am. J. Respir. Cell Mol. Biol. 25 (2001) 725-731; DOI: 10.1165/ajrcmb.25.6.4616.

9. S. Tamilvanan, Oil-in-water emulsions: implications for parenteral and ocular delivering systems, Prog. Lipid Res. 43 (2004) 489-533; DOI: 10.1016/j.plipres.2004.09.001.

10. N. A. Mazer, G. B. Benedek and M. C. Carey, Quasi elastic light-scattering studies of aqueous biliary lipid systems. Mixed micelle formation in bile salt-lecithin solutions, Biochemistry 19 (1980) 601-615; DOI: 10.1021/bi00545a001.

11. W. Mehnert and K. Mäder, Solid lipid nanoparticles: production, characterization and applications, Adv. Drug Deliv. Rev. 64 (2012) 83-101; DOI: 10.1016/j.addr.2012.09.021.

12. S. A. Wissing, O. Kayser and R. H. Müller, Solid lipid nanoparticles for parenteral drug delivery, Adv. Drug Deliv. Rev. 56 (2004) 1257-1272; DOI: 10.1016/j.addr.2003.12.002.

13. M. Brandl, Vesicular phospholipid gels: a technology platform, J. Liposome Res. 17 (2007) 15-26; DOI: 10.1080/08982100601186490.

14. M. Brandl, M. Drechsler, D. Bachmann, C. Tardi, M. Schmidtgen and K. H. Bauer, Preparation and characterization of semi-solid phospholipid dispersions and dilutions thereof, Int. J. Pharm. 170 (1998) 187-199; DOI: 10.1016/S0378-5173(98)00146-X.

15. C. Tardi, M. Drechsler, K. H. Bauer and M. Brandl, Steam sterilization of vesicular phospholipid gels, Int. J. Pharm. 217 (2001) 161-172; DOI: 10.1016/S0378-5173(01)00605-6.

16. M. Sznitowska, E. A. Dąbrowska and S. Janicki, Solubilizing potential of submicron emulsions and aqueous dispersions of lecithin, Int. J. Pharm. 246 (2002) 203-206; DOI: 10.1016/S03785173(02)00395-2.

17. M. Sznitowska, M. Klunder and M. Płaczek, Paclitaxel solubility in aqueous dispersions and mixed micellar solutions of lecithin, Chem. Pharm. Bull. 56 (2008) 70-74; DOI: 10.1248/cpb.56.70. 
18. M. Sznitowska, M. Bodnar, J. Petrusewicz, H. Janik and E. A. Dąbrowska, Preliminary in vivo studies of a new lecithin-based formulation of paclitaxel, J. Microencapsul. 26 (2009) 588-592; DOI: 10.3109/02652040802586068.

19. R. J. Haskell, Characterization of submicron systems via optical methods, J. Pharm. Sci. 87 (1998) 125-129; DOI: 10.1021/js970331i.

20. V. Klang, N. B. Matsko, C. Valenta and F. Hofer, Electron microscopy of nanoemulsions: an essential tool for characterisation and stability assessment, Micron 43 (2012) 85-103; DOI: 10.1016/j. micron.2011.07.014.

21. J. Kuntsche, J. C. Horst and H. Bunjes, Cryogenic transmission electron microscopy (cryo-TEM) for studying the morphology of colloidal drug delivery systems, Int. J. Pharm. 417 (2011) 120-137; DOI: 10.1016/j.ijpharm.2011.02.001.

22. S. Bibi, R. Kaur, M. Henriksen-Lacey, S. E. McNeil, J. Wilkhu, E. Lattmann, D. Christensen, A. R. Mohammed and Y. Perrie, Microscopy imaging of liposomes: from coverslips to environmental SEM, Int. J. Pharm. 417 (2011) 138-150; DOI: 10.1016/j.ijpharm.2010.12.021.

23. J. A. Litwin and M. Gajda, Podstawy technik mikroskopowych, Jagiellonian University Press, Cracow 2011.

24. P. E. West, Introduction to Atomic Force Microscopy, Pacific Nanotechnology, Santa Clara 2006, pp. $1-16$.

25. P. C. Schmidt, Secondary Electron Microscopy in Pharmaceutical Technology, in Encyclopedia of Pharmaceutical Technology (Ed. J. Swarbrick), Informa Healthcare, New York 2007, pp. 3217-3256.

26. K. Akashi, H. Miyata, H. Itoh and K. Kinosita, Formation of giant liposomes promoted by divalent cations: critical role of electrostatic repulsion. Biophys. J. 74 (1998) 2973-2982; DOI: 10.1016/S00063495(98)78004-X.

27. R. M. Fernandez, K. A. Riske, L. Q. Amaral, R. Itri and M. T. Lamy, Influence of salt on the structure of DMPG studied by SAXS and optical microscopy, Biochim. Biophys. Acta. 1778 (2008) 907916; DOI: 10.1016/j.bbamem.2007.12.005.

28. C. C. Müller-Goymann, Physicochemical characterization of colloidal drug delivery systems such as reverse micelles, vesicles, liquid crystals and nanoparticles for topical administration, Eur. J. Pharm. Biopharm. 58 (2004) 343-356; DOI: 10.1016/j.ejpb.2004.03.028.

29. C. C. Müller-Goymann, Drug Delivery: Liquid Crystals, in Encyclopedia of Pharmaceutical Technology (Ed. J. Swarbrick), Informa Healthcare, New York 2007, pp. 1115-1131.

30. A. Graf, E. Ablinger, S. Peters, A. Zimmer, S. Hook and T. Rades, Microemulsions containing lecithin and sugar-based surfactants: nanoparticle templates for delivery of proteins and peptides, Int. J. Pharm. 350 (2008) 351-360; DOI: 10.1016/j.ijpharm.2007.08.053.

31. N. Rodriguez, F. Pincet and S. Cribier, Giant vesicles formed by gentle hydration and electroformation: a comparison by fluorescence microscopy, Colloids Surf. B. Biointerfaces 42 (2005) 125-130; DOI: 10.1016/j.colsurfb.2005.01.010.

32. P. Arunothayanun, M. S. Bernard, D. Q. M. Craig, I. F. Uchegbu and A. T. Florence, The effect of processing variables on the physical characteristics of non-ionic surfactant vesicles (niosomes) formed from a hexadecyl diglycerol ether, Int. J. Pharm. 201 (2000) 7-14; DOI: 10.1016/S03785173(00)00362-8.

33. S. R. Pygall, J. Whetstone, P. Timmins and C. D. Melia, Pharmaceutical applications of confocal laser scanning microscopy: the physical characterization of pharmaceutical systems, Adv. Drug Deliv. Rev. 59 (2007) 1434-1452; DOI: 10.1016/j.addr.2007.06.018

34. J. Ch. Colas, W. Shi, V. S. Rao, A. Omri, M. R. Mozafari and H. Singh, Microscopical investigations of nisin-loaded nanoliposomes prepared by Mozafari method and their bacterial targeting, $\mathrm{Mi}^{-}$ cron 38 (2007) 841-847; DOI: 10.1016/j.micron.2007.06.013. 
35. B. Ruozi, D. Belletti, A. Tombesi, G. Tosi, L. Bondioli, F. Forni and M. A. Vandelli, AFM, ESEM, TEM, and CLSM in liposomal characterization: a comparative study, Int. J. Nanomedicine 6 (2011) 557-563; DOI: 10.2147/IJN.S14615.

36. M. A. Schubert and C. C. Müller-Goymann, Characterisation of surface-modified solid lipid nanoparticles (SLN): influence of lecithin and nonionic emulsifier, Eur. J. Pharm. Biopharm. 61 (2005) 77-86; DOI: 10.1016/j.ejpb.2005.03.006.

37. H. Zhou, Y. Yue, G. Liu, Y. Li, J. Zhang, Q. Gong, Z. Yan and M. Duan, Preparation and characterization of a lecithin nanoemulsion as a topical delivery system, Nanoscale Res. Lett. 5 (2010) 224230; DOI: 10.1007/s11671-009-9469-5.

38. S. A. Abraham, K. Edwards, G. Karlsson, S. MacIntosh, L. D. Mayer, C. McKenzie and M. B. Bally, Formation of transition metal-doxorubicin complexes inside liposomes, Biochim. Biophys. Acta 1565 (2002) 41-54; DOI: 10.1016/S0005-2736(02)00507-2.

39. M. Ciobanu, B. Heurtault, P. Schultz, C. Ruhlmann, C. D. Muller and B. Frisch, Layersome: development and optimization of stable liposomes as drug delivery system, Int. J. Pharm. 344 (2007) 54-57; DOI: 10.1016/j.ijpharm.2007.05.037.

40. H. Teixeira, C. Dubernet, V. Rosilio, S. Benita, J. Lepault, I. Erk and P. Couvreur, New bicompartmental structures are observed when stearylamine is mixed with triglyceride emulsions, Pharm. Res. 17 (2000) 1329-1332; DOI: 10.1023/A:1026416208482.

41. K. Jores, W. Mehnert, M. Drechsler, H. Bunjes, C. Johann and K. Mäder, Investigations on the structure of solid lipid nanoparticles (SLN) and oil-loaded solid lipid nanoparticles by photon correlation spectroscopy, field-flow fractionation and transmission electron microscopy, J. Control. Release 95 (2004) 217-227; DOI: 10.1016/j.jconrel.2003.11.012

42. A. Graf, E. Ablinger, S. Peters, A. Zimmer, S. Hook and T. Rades, Microemulsions containing lecithin and sugar-based surfactants: nanoparticle templates for delivery of proteins and peptides, Int. J. Pharm. 350 (2008) 351-360; DOI: 10.1016/j.ijpharm.2007.08.053.

43. G. De Rosa, M. De Stefano, F. Ungaro and M. I. La Rotonda, Cold field emission gun-scanning electron microscopy: a new tool for morphological and ultrastructural analysis of liposomes, Int. J. Pharm. 362 (2008) 189-192; DOI: 10.1016/j.ijpharm.2008.06.003.

44. A. Saupe, K. C. Gordon and T. Rades, Structural investigations on nanoemulsions, solid lipid nanoparticles and nanostructured lipid carriers by cryo-field emission scanning electron microscopy and Raman spectroscopy, Int. J. Pharm. 314 (2006) 56-62; DOI: 10.1016/j.ijpharm.2006.01.022.

45. N. G. Eskandar, S. Simovic and C. A. Prestidge, Nanoparticle coated submicron emulsions: sustained in-vitro release and improved dermal delivery of all-trans-retinol, Pharm. Res. 26 (2009) 1764-1775; DOI: 10.1007/s11095-009-9888-0.

46. Y. Perrie, A. U. Mohammed, A. Vangala and S. E. McNeil, Environmental scanning electron microscopy offers real-time morphological analysis of liposomes and niosomes, J. Liposome Res. 17 (2007) 27-37; DOI: 10.1080/08982100601186508.

47. A. Bogner, G. Thollet, D. Basset, P. H. Jouneau and C. Gauthier, Wet STEM: a new development in environmental SEM for imaging nano-objects included in a liquid phase, Ultramicroscopy 104 (2005) 290-301; DOI: 10.1016/j.ultramic.2005.05.005.

48. J. Sitterberg, A. Özcetin, C. Ehrhardt and U. Bakowsky, Utilising atomic force microscopy for the characterisation of nanoscale drug delivery systems, Eur. J. Pharm. Biopharm. 74 (2010) 2-13; DOI: 10.1016/j.ejpb.2009.09.005.

49. B. Ruozi, G. Tosi, E. Leo and M. A. Vandelli, Application of atomic force microscopy to characterize liposomes as drug and gene carriers, Talanta 73 (2007) 12-22; DOI: 10.1016/j.talanta.2007.03.031.

50. X. Liang, G. Mao and K. Y. Ng, Mechanical properties and stability measurement of cholesterolcontaining liposome on mica by atomic force microscopy, J. Colloid Interface Sci. 278 (2004) 53-62; DOI: $10.1016 /$ j.jcis.2004.05.042. 
51. C. Preetz, A. Hauser, G. Hause, A. Kramer and K. Mäder, Application of atomic force microscopy and ultrasonic resonator technology on nanoscale: distinction of nanoemulsions from nanocapsules, Eur. J. Pharm. Sci. 39 (2010) 141-151; DOI: 10.1016/j.ejps.2009.11.009.

52. T. Tran, T. C. Kupiec and L. A. Trissel, Quality-control analytical methods: particulate matter in injections: what is it and what are the concerns?, Int. J. Pharm. Compd. 10 (2006) 202-204.

53. S. E. Langille, Particulate matter in injectable drug products, PDA J. Pharm. Sci. Technol. 67 (2013) 186-200; DOI: 10.5731/pdajpst.2013.00922.

54. Ch. M. Hoo, N. Starostin, P. West and M. L. Mecartney, A comparison of atomic force microscopy (AFM) and dynamic light scattering (DLS) methods to characterize nanoparticle size distributions, J. Nanopart. Res. 10 (2008) 89-96; DOI: 10.1007/s11051-008-9435-7.

55. H. Kato, A. Nakamura and N. Noda, Determination of size distribution of silica nanoparticles: a comparison of scanning electron microscopy, dynamic light scattering, and flow-field-flow fractionation with multiangle light scattering methods, Mater. Express 4 (2014) 144-152; DOI: 10.1166/ mex.2014.1150.

56. V. Klang, C. Valenta and N. B. Matsko, Electron microscopy of pharmaceutical systems, Micron 44 (2013) 45-74; DOI: 10.1016/j.micron.2012.07.008. 